\title{
ANOTHER VIEW OF METRIZABILITY
}

\author{
H. H. HUNG
}

(Communicated by Dennis Burke)

\begin{abstract}
A fact long considered unsatisfactory about the classical metrization theorem of Alexandroff-Urysohn is that it expresses metrizability as a countable uniformity, uniformity itself being almost the former. In view of their unification, the classical theorems, with the exception of Arhangel'skir's regular open base theorem, are all really subject to the same criticism, to which our theorem here is an answer. We give a generalization here of Arhangel'skiris, of which Arhangel'skir's itself, the fundamental theorem of Alexandroff-Urysohn, A. H. Frink's, and the Double Sequence Theorem of Nagata are all obvious special cases.
\end{abstract}

Except for the regular open base of Arhangel'skil [2] (Theorem VI. 7 of [18]), common to all ${ }^{1}$ metrizing bases, in the absence of extensive assumption of separation axioms, such as paracompactness or collectionwise normality, is the idea of uniformity: the metrizing base in each case is made up of a sequence of collections, separately imposed on each of which are conditions that refer little if at all to collections beyond the next. This fact is most obvious in Nagata's systematic account of them [17, 18], in Hodel's unified approach in their treatment [10], and in my unification of them into particular cases of one master theorem [11]. Yet, it was precisely because of this uniformity component in Alexandroff and Urysohn [1] and in A. H. Frink [8] that these theorems were regarded as unsatisfactory topological metrization theorems at the time (see e.g. $\S 14$ of Chapter 6 of Kelley [14]). In any case, the requirement that the metrizing base can be written as a union of countably many collections before topological conditions are imposed on each of them individually is no small requirement and, in this light, Arhangel'skiı̀'s regular open base theorem is singularly attractive. Here we are to generalize Arhangel'skir to the extent that we have as mere special cases Alexandroff and Urysohn [1], still so fundamental in the theory in the opinion of Nagata (see p. 245 of [18]), along with Frink [8], and Nagata's Double Sequence Theorem [17], from the former of which follows easily the latter as do almost all others, according to Martin [15]. We have thus arrived at a new point of view and more unity among the theorems,

Received by the editors July 30, 1985 and, in revised form, November 8, 1985, March 25, 1986, and July 28, 1986. Presented to the Society in different formulations on December 24, 1984, November 4, 1985 and March 19, 1986.

1980 Mathematics Subject Classification (1985 Revision). Primary 54E35, 54F65.

Key words and phrases. Metrizability without uniformity among members of bases, pairnetworks, nests, companions of nests, capture of nests by their companions.

${ }^{1}$ A partial exception is the main theorem of Collins, Reed, Roscoe, and Rudin [6], announced in Collins and Roscoe [7]. But even there, the local bases $W(N, x), x \in X$, are required to fulfill the condition that $\xi \notin \mathrm{Cl}\{x \in X: \xi \notin W(1, x)\}$ for any $\xi \in X$, one of uniformity. The main theorem of Collins and Roscoe is no exception at all and is in fact part of Corollary 2.4 of [11]. 
having been inspired by Arhangel'skiu [2, 3], by Collins, Reed, Roscoe, and Rudin [6], and by Gruenhage and Zenor [9].

DEFINITIONS. On a $T_{1}$ space $X$, a pair-network (cf. [4]) is a collection $A$ of pairs $\left\{A, A^{\prime}\right\}$ of sets, $A^{\prime}$ being open and nonvoid and contained in $A$, such that, for any neighborhood $U$ of any $x \in X$, there is such a $\left\{B, B^{\prime}\right\} \in A$ that $x \in B^{\prime} \subset$ $B \subset U$. A nest $B$ is a well-ordered, infinite subcollection of $A$ such that, of $B$, $\left\{A, A^{\prime}\right\}<\left\{B, B^{\prime}\right\} \Rightarrow A^{\prime} \supsetneqq B$. If $\bigcap\left\{A^{\prime}:\left\{A, A^{\prime}\right\} \in B\right\}$ is nonvoid (and contains $\xi$ ) we say $B$ is fixed (at $\xi$ ). Given a nest $B$, its first element $\left\{I, I^{\prime}\right\}$; if there is, for every $\left\{B, B^{\prime}\right\} \in B$, one $\left\{\hat{B}, \hat{B}^{\prime}\right\} \in A$ such that $\hat{B}^{\prime} \cap B^{\prime} \neq \varnothing$ and $\hat{B}^{\prime} \backslash I \neq \varnothing$, we say $C \equiv\left\{\left\{\hat{B}, \hat{B}^{\prime}\right\}:\left\{B, B^{\prime}\right\} \in B\right\}$ is a companion of $B$. We further say that $C$ captures $B$ if $\bigcup\left\{C:\left\{C, C^{\prime}\right\} \in C\right\} \supset B$ for some $B \in B$.

THEOREM. A $T_{1}$ space is metrizable if (and only if) there is on it such a pairnetwork that every fixed nest in it is

(i) (order) isomorphic to $\omega$, and

(ii) captured by each of its companions.

EXAMPLE 1. Any system of pairs of concentric balls of radii ratios greater than some $\alpha>1$, numerous enough to form a pair-network, is such a pair-network.

From this example follows the necessity of the conditions of the Theorem for metrizability.

EXAMPLE 2. In a regular open base $R$ of Arhangel'skir [2], there is such a pairnetwork in $\mathcal{A} \equiv\left\{\left\{R, R^{\prime}\right\}: R, R^{\prime} \in R\right.$, only finitely many members of $R$ intersect both $\sim R$ and $\left.R^{\prime}\right\}$.

For, for any nest $B \subset A$, any companion $C$ of $B$ is necessarily finite and at least one of these finitely many members, $\left\{C, C^{\prime}\right\}$, is such that $C \supset B$ for some $\left\{B, B^{\prime}\right\} \in B$.

Arhangel'skiî's Theorem is therefore a special case of our theorem.

EXAMPLE 3. Among the open sets in every sequence $\left\{U_{i}: i \in \mathbf{N}\right\}$ of open covers described in the metrization theorem of Alexandroff and Urysohn [1], as it appears as Theorem VI.1 of [18], there is such a pair-network in

$$
\mathcal{A} \equiv\left\{\left\{U, U^{\prime}\right\}: U \in \mathcal{U}_{i}, U^{\prime} \in \mathcal{U}_{i+1}, \operatorname{St}\left(U^{\prime}, U_{i+1}\right) \subset U, i \in \mathbf{N}\right\} .
$$

Alexandroff and Urysohn is therefore a special case of our theorem.

EXAMPLE 4. Among the neighborhood bases $\left\{V_{n}(x): n \in \mathbf{N}\right\}, x \in X$, assumed decreasing without loss of generality, in Frink's metrization theorem, as it appears in [15], there is such a pair-network in

$$
\mathcal{A} \equiv\left\{\left\{V_{n}(x), \operatorname{Int} V_{m(x, n)}(x)\right\}: n \in \mathbf{N}, x \in X\right\} .
$$

One notes that, for all $x, \xi \in X$ and $n, \nu \in \mathbf{N}$, Int $V_{m(x, n)}(x) \cap \operatorname{Int} V_{m(\xi, \nu)}(\xi) \neq \varnothing$ and $m(x, n) \leq m(\xi, \nu) \Rightarrow V_{n}(x) \supset$ Int $V_{m(\xi, \nu)}(\xi)$.

Frink's Theorem is therefore a special case of ours.

EXAMPLE 5. Among the sequences of neighborhoods of Nagata's Double Sequence Theorem (Theorem VI.2 of [18]), assumed decreasing without loss of generality, there is such a pair-network in

$$
\begin{aligned}
A \equiv\left\{\left\{V_{m}(x), \text { Int } V_{n}(x)\right\}: m, n \in \mathbf{N}, x \in X,\right. \\
\left.V_{m}(x) \supset U_{k}(x) \supset V_{k}(x) \supset U_{n}(x) \supset V_{n}(x), \text { for some } m<k<n\right\} .
\end{aligned}
$$


Nagata's Theorem is therefore also a special case of ours.

Note from the above that while pair-networks of the description of the Theorem are readily discerned in the structures of Arhangel'skil., Alexandroff and Urysohn, Frink, and Nagata, it is difficult if not impossible to see in our pair-network any of these structures. It seems therefore we have not only what is common in the four structures, but also something weaker than any of the four.

PROOF OF THE THEOREM. To prove that any $T_{1}$ space $X$ on which can be defined such a system $A$ is metrizable, it suffices to prove that $X$ is paracompact (Hausdorff) and has a BCO $[3,12,20]$. In the following, we write $(x, U)$ for $\left\{\left\{A, A^{\prime}\right\} \in A: x \in A^{\prime} \subset A \subset U\right\}$ for every neighborhood $U$ of $x \in X$. By definition, $(x, U) \neq \varnothing$.

We first prove that $(*)$ for any nest $B$ fixed at $\xi$, the set $B^{\prime} \equiv\left\{A^{\prime}:\left\{A, A^{\prime}\right\} \in B\right\}$ is a local base at $\xi$, that is to say that, given any open neighborhood $V$ of $\xi$ and any $\left\{C, C^{\prime}\right\} \in(\xi, V), C$ (and therefore $\left.V\right)$ contains $B^{\prime}$ for some $\left\{B, B^{\prime}\right\} \in B$. Since $\cap B^{\prime}$ is not a neighborhood of $\xi$, we have $D \nsupseteq C^{\prime}$, i.e., $C^{\prime} \backslash D \neq \varnothing$ for some $\left\{D, D^{\prime}\right\} \in B$ and $\left\{\left\{C, C^{\prime}\right\}\right\}$ is a companion of the nest $\left\{\left\{A, A^{\prime}\right\} \in B:\left\{D, D^{\prime}\right\} \leq\left\{A, A^{\prime}\right\}\right\}$. There is therefore $\left\{B, B^{\prime}\right\} \in B$ such that $C \supset B \supset B^{\prime}$.

To prove that $X$ has a BCO, we need construct only an $(m d)$-sieve [5]. Here we describe the $(m d)$-sieve as a tree ${ }^{2} T$ of $\omega$-levels of nonvoid open sets arranged in such a way that

(i) $\bigcup T=X$,

(ii) $a=\bigcup\{b: a<b\}$ for all $a \in T$, and

(iii) if $B \subset T$ is a branch and if $\xi \in \bigcap B$, then $B$ is a local base at $\xi$.

Clearly, such a tree would have been defined if we let the first level of it be the collection $\left\{A^{\prime}:\left\{A, A^{\prime}\right\} \in A\right\}$ and for every nonsingleton $M$ on $T$, let the set of its successors be $\left\{A^{\prime}:\left\{A, A^{\prime}\right\} \in A, A \varsubsetneqq M\right\}$, provided (iii) is proved, i.e., provided proved is that $(\mathrm{\dagger})$ a branch $B$ of nonsingeltons is a local base at $\xi$ if $\xi \in \bigcap B$. But then, any such a branch corresponds to a nest $B$ in $A$, and (*) above is equivalent to $(t)$.

To prove that $X$ is paracompact (Hausdorff), we make use of Theorem 3 of [13] and let $\mathcal{U}$ be a well-ordered open cover of $X$. Let $A_{x} \equiv \bigcup\{(x, U): U \in \mathcal{U}\}$ for every $x \in X$. Define a maximal well-ordered discrete subset $Y \subset X$ such that, of $Y, y<z \Rightarrow z \notin \bigcup\left\{A^{\prime}:\left\{A, A^{\prime}\right\} \in A_{y}\right\}$. We write $A_{Y}$ for $\bigcup\left\{A_{x}: x \in Y\right\}$. Clearly $\left\{A^{\prime}:\left\{A, A^{\prime}\right\} \in A_{Y}\right\}$ is (a cover of $X$ and) a refinement of $\mathcal{U}$. For each $U \in \mathcal{U}$, let $A_{Y, U} \equiv\left\{\left\{A, A^{\prime}\right\} \in A_{Y}: A \subset U, A \not \subset V<U\right\}$ (partitioning $A_{Y}$ ). Clearly, if $\left\{A, A^{\prime}\right\} \in A_{Y, U}$, then $\left\{A, A^{\prime}\right\} \in A_{x}$ for some $x \in U \cap Y$. Let $A_{Y, U}^{\prime}$ be $\left\{A^{\prime}:\left\{A, A^{\prime}\right\} \in A_{Y, U}\right\}$.

Now we are ready to define a function $f$ on $U$ into the topology of $X$ as follows. For all $U \in \mathcal{U}$, let $f(U) \equiv \bigcup A_{Y, U}^{\prime}$. Clearly, (i) $f(U) \subset U$ for all $U \in \mathcal{U}$, and (ii) $f[U]$ covers $X$. Clear also is the fact that, for all $U \in \mathcal{U}$, (iii) $\operatorname{Cl} \cup\{f(V): V<U\} \subset$ $\bigcup\{V: V<U\}$. For, otherwise, there is $\xi \in \operatorname{Cl} \bigcup\{f(V): V<W\} \backslash \bigcup\{V: V<W\}$ for some $W \in \mathcal{U}$ and while there certainly are nests $B$ fixed at $\xi$ that begin with such an $\left\{I, I^{\prime}\right\}$ that $I$ is disjoint from $\bigcup\{V \cap Y: V<W\}$, they are never captured by any companion $C$ built of elements of $\bigcup\left\{A_{Y, V}: V<W\right\}$, a contradiction.

\footnotetext{
${ }^{2}$ Note that we can have the same open set occupying more than one position on $T$, i.e., we can have $a, b \in T, a \neq b$ as members of $T$ and yet $a=b$ as open sets of $X$.
} 
REMARK. That (i) in our metrizing conditions is necessary can be seen in the example of the Michael line [16], with a natural pair-network satisfying (ii) obviously and failing to satisfy (i) equally obviously. In contrast, the Sorgenfrey line [19] has one that satisfies (i) but not (ii).

\section{REFERENCES}

1. P. S. Alexandroff and P. Urysohn, Une condition necessaire et suffisante pour qu'une class $(\mathcal{L})$ soit une class (D), C. R. Acad. Sci. Paris 177 (1923), 1274-1277.

2. A. V. Arhangel'skiř, On the metrization of topological spaces, Bull. Acad. Polon. Math. Ser. 8 (1960), 589-595. (Russian)

3. __ Some metrization theorems, Uspehi Mat. Nauk 18 (1963), no. 5 (113), 139-145. (Rus$\operatorname{sian)}$

4. D. K. Burke, Pseudo-open mappings from topological sums, Proc. Amer. Math. Soc. 74 (1979), 191-196.

5. J. Chaber, M. M. Coban, and K. Nagami, On monotonic generalizations of Moore spaces, Cech complete spaces and p-spaces, Fund. Math. 84 (1974), 107-119.

6. P. J. Collins, G. M. Reed, A. W. Roscoe, and M. E. Rudin, A lattice of conditions on topological spaces, Proc. Amer. Math. Soc. 94 (1985), 487-496.

7. P. J. Collins and A. W. Roscoe, Criteria for metrisability, Proc. Amer. Math. Soc. 90 (1984), 631-640.

8. A. H. Frink, Distance functions and the metrization problem, Bull. Amer. Math. Soc. 43 (1937), 133-142.

9. G. Gruenhage and P. Zenor, Proto-metrizable spaces, Houston J. Math. 3 (1977), 47-53.

10. R. Hodel, Spaces defined by sequences of open covers which guarantee that certain sequences have cluster points, Duke Math. J. 39 (1972), 253-263.

11. H. H. Hung, A contribution to the theory of metrization, Canad. J. Math. 29 (1977), 1145-1151.

12. __ On a theorem of Arhangel'skii, Proc. Amer. Math. Soc. 82 (1981), 629-633.

13. _, A refinement on Michael's characterization of paracompactness, Proc. Amer. Math. Soc. 83 (1981), 179-182.

14. J. L. Kelley, General topology, Van Nostrand, Princeton, N. J., 1955.

15. H. W. Martin, A note on the Frink metrization theorem, Rocky Mountain J. Math. 6 (1976), 155-157.

16. E. Michael, The product of a normal space and a metric space need not be normal, Bull. Amer. Math. Soc. 69 (1963), 375-376.

17. J. Nagata, A contribution to the theory of metrization, J. Inst. Polytech. Osaka City Univ. 8 (1957), 185-192.

18. , Modern general topology, 2nd rev. ed., North-Holland, Amsterdam, 1985.

19. R. H. Sorgenfrey, On the topological product of paracompact spaces, Bull. Amer. Math. Soc. 53 (1947), 631-632.

20. J. M. Worrell Jr. and H. H. Wicke, Characterizations of developable topological spaces, Canad. J. Math. 17 (1965), 820-830.

Department of Mathematics, Concordia University, Montréal, Québec, CANADA H4B 1R6 